\title{
Effect of prophylactically administered edaravone during antegrade cerebral perfusion in a canine model of old cerebral infarction
}

Takayasu Suzuki, MD, ${ }^{\text {a }}$ Teruhisa Kazui, MD, PhD, ${ }^{\text {a }}$ Seiji Yamamoto, MD, PhD, ${ }^{\text {b }}$ Naoki Washiyama, MD, PhD, ${ }^{a}$ Kazuhiro Ohkura, MD, PhD, ${ }^{a}$ Kentaro Ohishi, PhD, ${ }^{\text {b }}$ Abul Hasan Muhammad Bashar, MBBS, PhD, ${ }^{\text {a }}$ Katsushi Yamashita, MD, PhD, ${ }^{a}$ Hitoshi Terada, MD, PhD, ${ }^{a}$ Kazuchika Suzuki, MD, PhD, ${ }^{a}$ Satoshi Akuzawa, MD, ${ }^{a}$ and Michio Fujie, $\mathrm{MS}^{\mathrm{c}}$

From the First Department of Surgery, ${ }^{\mathrm{a}}$ Photon Medical Research Center, ${ }^{\mathrm{b}}$ and Research Equipment Center, ${ }^{\mathrm{c}}$ Hamamatsu University School of Medicine, Hamamatsu, Japan.

Received for publication Aug 10, 2006; revisions received Oct 9, 2006; accepted for publication Oct 30, 2006.

Address for reprints: Takayasu Suzuki, MD, The First Department of Surgery, Hamamatsu University School of Medicine, 1-20-1 Handayama, Hamamatsu, 4313192, Japan (E-mail: tac.sz@hama-med. ac.jp).

J Thorac Cardiovasc Surg 2007;133:710-6

$0022-5223 / \$ 32.00$

Copyright $\odot 2007$ by The American Association for Thoracic Surgery

doi:10.1016/j.jtcvs.2006.10.032
Objective: Reactive free radical species are thought to be involved in postoperative neurologic dysfunction after antegrade selective cerebral perfusion in brains with old infarction. We assessed the brain protective effect of prophylactically administered edaravone, a free radical scavenger, for antegrade selective cerebral perfusion in brains with or without old infarction in a canine model.

Methods: A canine model of old cerebral infarction was created by injecting cylindric silicone embolus into the middle cerebral artery. Animals showing obvious neurologic deficits and surviving 4 weeks or longer were included in the model. Deep hypothermia with antegrade selective cerebral perfusion was performed in both intact (non-edaravone, group A; edaravone-treated, group B) and infarcted animals (non-edaravone, group C; edaravone-treated, group D). Serum concentrations of malondialdehyde, hexanoyl-lysine, glutamate, and venous-arterial lactate difference were measured, and central conduction time and amplitude of somatosensory evoked potentials were assessed during the operation.

Results: Compared with the intact groups, serum concentrations of malondialdehyde and hexanoyl-lysine in group $\mathrm{C}$ significantly increased at the end of antegrade selective cerebral perfusion, whereas that of glutamate did so in the rewarming phase. Increases in all these biochemical parameters were suppressed in group D. In group $\mathrm{C}$, the venous-arterial lactate difference was significantly greater in the rewarming phase at $28^{\circ} \mathrm{C}$ compared with intact groups. A significant prolongation of postoperative central conduction time and decrease in neuronal activity were detected in group $\mathrm{C}$, both of which recovered in group D.

Conclusion: Prophylactic administration of edaravone exerted a significant protective effect against postoperative neurologic dysfunction after antegrade selective cerebral perfusion in a canine model with old cerebral infarction.

$\mathrm{P}$ ostoperative neurologic dysfunction is one of the most serious complications after aortic arch operation. The incidence of this complication has decreased in recent times because of improvements in operative techniques and cerebral protection methods. ${ }^{1}$ Antegrade selective cerebral perfusion (ASCP) has been found to be the safest method of brain protection during aortic arch surgery with respect to energy metabolism and time limitation. ${ }^{2,3}$ Clinical practice has indicated that ASCP can reduce cerebral injury during aortic arch operation more effectively compared with other methods for cerebral protection, including deep hypothermic circulatory arrest with or without retrograde cerebral perfusion. ${ }^{4}$ Although our total arch replacement assisted with ASCP has resulted in a lower rate of mortality and morbidity, ${ }^{5}$ a multivariable analysis of 220 patients revealed that a history of 


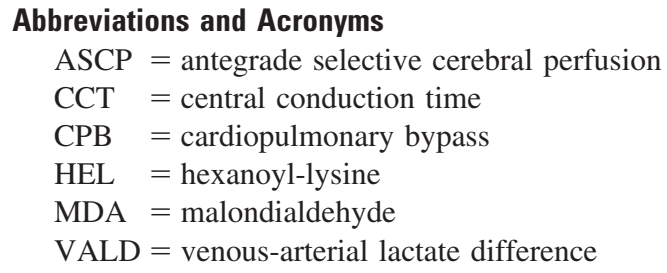

cerebral infarction should be regarded as an independent predictor of postoperative neurologic dysfunction. ${ }^{6}$

Our previous canine studies showed that ASCP in animals with old cerebral infarction induced ischemic neurologic damage and that it increased the production of free radicals, suggesting oxidative damage in the peri-infarct region of the brain. ${ }^{7,8}$ Many previous reports described accelerated production of free radicals in acute cerebral infarction resulting in neurologic damage, and free radical scavenger has already been found to be effective in attenuating such neurologic damage in clinical situations. ${ }^{9,10}$ Similarly, a recent experimental study from our laboratory documented that edaravone (Mitsubishi Pharma Co, Tokyo, Japan), a commercially available free radical scavenger, was effective in attenuating acute ischemic neurologic damage in the rabbit spinal cord. ${ }^{11}$ However, the exact relationship between free radicals and old cerebral infarction is unknown. A pharmacologic agent with a similar protective effect on brains with old cerebral infarct can be of great clinical relevance during an aortic arch operation with ASCP. Therefore, in the present study, we assessed the neuroprotective effect of prophylactically administered edaravone for ASCP in brains with or without old cerebral infarction in a canine model.

\section{Materials and Methods}

The study was approved by the Animal Care and Use Committee of Hamamatsu University School of Medicine. All animals received human care in compliance with the "Guide for the Care and Use of Laboratory Animals," published by the National Institutes of Hearth (NIH publication 85-23, revised 1985), and the "Guidelines for Animal Experimentation," formulated by Hamamatsu University School of Medicine (published 1987, revised 1991).

\section{Cerebral Infarct Model}

Our technique of creating a cerebral infarct model has been described. ${ }^{7}$ In short, the left common carotid artery with its bifurcation was surgically exposed, and a cylindric silicone embolus was injected into the left internal carotid artery. Nonvisualization of the left middle cerebral artery in the subsequent angiogram suggested that the embolus was located in the proximal left middle cerebral artery. Twelve hours later, the dogs were evaluated with a neurologic scoring system consisting of 5 grades (score 0 : no neurologic deficit; score 1: walks with limp or circles to the side of lesion; score 2: walks poorly and stands but cannot support the body with a left limb held off the ground; score 3: cannot stand without support; and score 4: dead). ${ }^{12}$ Only the dogs with neurologic scores of 2 and 3, and surviving 4 weeks or longer were included in the cerebral infarct model.

\section{Animal Preparation}

After general anesthesia with an intravenous injection of pentobarbital sodium (30 mg/kg), mechanical ventilation was started. A 19-gauge detaining needle was inserted into the right brachial artery for blood sampling and blood pressure monitoring. A 4F catheter was placed into the right maxillary vein for sampling of venous blood for blood gas analysis and lactate measurement. An additional dose of pentobarbital sodium $(15 \mathrm{mg} / \mathrm{kg})$ was given before the beginning of rewarming.

\section{Cardiopulmonary Bypass}

After median sternotomy and full heparinization (300 U/kg), cardiopulmonary bypass (CPB) was instituted by inserting the arterial cannula into the ascending aorta, and the venous cannulae into the superior and inferior vena cavae. The perfusion system, consisting of a roller pump and membrane oxygenator (Senkoika Corp, Tokyo, Japan), was primed with lactated Ringer solution. A second dose of heparin $(150 \mathrm{U} / \mathrm{kg})$ was added before the beginning of rewarming. Alpha-stat strategy was used for blood $\mathrm{pH}$ management.

\section{Measurement of Somatosensory Evoked Potentials}

Single-channel recordings were obtained on a Nicolet Compact Four/CA 2000 (Nicolet JAPAN Corp, Tokyo, Japan). Three needle electrodes were positioned: the first over the head approximately 2 $\mathrm{cm}$ dorsal to the coronal suture, the second in the upper right-hand side of the sternum, and the third (the reference electrode) on the right shoulder. The median nerve of the right forelimb was exposed and stimulated with a bipolar stimulator. The pulse duration used was $100 \mathrm{msec}$, and the strength of the stimulating current was 2 to $5 \mathrm{~mA}$ with a frequency of $5.1 \mathrm{~Hz}$. A total of 300 responses were obtained and averaged with automatic artifact rejection by setting the filter between approximately 5 and $1000 \mathrm{~Hz}$. The central conduction time (CCT), indicating the transmission time of neuronal activity in the brain, was measured as an interpeak latency of $\mathrm{N} 2$ (the second negative wave) and N4 (the fourth negative wave). The amplitude ratio between N4 and N1 (the first negative wave), an indicator of neuronal activity in the sensory cortex, was calculated. The preoperative and postoperative values were compared.

\section{Experimental Protocol}

Twenty-four female mongrel dogs were divided into the following 4 groups: group $\mathrm{A}$, intact, non-edaravone group $(\mathrm{n}=6)$; group $\mathrm{B}$, intact, edaravone-treated group $(n=6)$; group $C$, infarct, nonedaravone group $(n=6)$; and group $D$, infarct, edaravone-treated group $(n=6)$. Animals of all 4 groups underwent deep hypothermia with ASCP. CPB flow was maintained at approximately 50 to $80 \mathrm{~mL} / \mathrm{kg} / \mathrm{min}$ in accordance with the amount of venous return. After the animals were cooled to a rectal temperature of $20^{\circ} \mathrm{C}$, ASCP was initiated at a flow rate of $10 \mathrm{~mL} / \mathrm{kg} / \mathrm{min}$ by clamping the proximal ascending aorta, the left subclavian artery, and the descending aorta. The lower half of the body was not perfused 
during ASCP. After 120 minutes of ASCP, CPB was returned to its original flow rate. Then, rewarming to a rectal temperature of $36^{\circ} \mathrm{C}$ was performed. Differences between the rectal and arterial temperatures were always kept within $5^{\circ} \mathrm{C}$ in both cooling and rewarming phases.

\section{Drug Delivery Protocol}

In the edaravone-treated groups, first, edaravone $1.5 \mathrm{mg} / \mathrm{kg}$ in 100 $\mathrm{mL}$ normal saline solution was dripped into the extracorporeal circuit just before the beginning of cooling. Because it takes approximately 30 minutes to obtain a steady plasma level of edaravone after its intravenous injection, we made sure that the first dose of edaravone was completed 30 minutes before the initiation of ASCP. ${ }^{13}$ To ensure a stable plasma level of the drug until the end of the experiment, a second dose of edaravone (1.5 $\mathrm{mg} / \mathrm{kg}$ ) was added to the circuit in the same method 30 minutes after the first. For the non-edaravone group, only $100 \mathrm{~mL}$ of normal saline solution without edaravone was administered into the circuit at the same time points.

\section{Protocol for Blood Biochemistry}

Blood samples were obtained on the following 8 occasions: (1) before the operation; (2) 5 minutes after the initiation of CPB; (3) on reaching a rectal temperature of $28^{\circ} \mathrm{C}$ in the cooling phase; (4) on reaching a rectal temperature of $20^{\circ} \mathrm{C}$ in the cooling phase; (5) 60 minutes after the initiation of ASCP; (6) at the end of ASCP; (7) on reaching a rectal temperature of $28^{\circ} \mathrm{C}$ in the rewarming phase; and (8) on reaching a rectal temperature of $36^{\circ} \mathrm{C}$ in the rewarming phase. Sampled blood was centrifuged, and protein was extracted from the serum. Malondialdehyde (MDA), a marker of free radical production and lipid peroxidation of membrane polyunsaturated fatty acid, was measured by means of thiobarbituric acid fluorescence (FP-777; Nihon Bunko, Tokyo, Japan). ${ }^{14,15}$ Hexanoyl-lysine (HEL) formed by the reaction between linoleic acid hydroperoxide and lysine is another biomarker of oxidative stress, which was measured by the enzyme-linked immunosorbent assay method using the HEL adduct enzyme-linked immunosorbent assay kit (Japan Institute for the Control of Aging, Shizuoka, Japan). ${ }^{16,17}$ Serum glutamate, a biomarker of neural cell damage, was measured on a column packed with reverse-phase support with a special device (PICO-TAG; Waters Corp, Milford, Mass). Blood lactate was measured using a blood gas analyzer (Stat Profile Ultra M; Nova Biomedical Corp, Tokyo, Japan), and the venous-arterial lactate difference (VALD) was calculated.

\section{Statistical Analysis}

All values are expressed as means \pm standard error. One-way analysis of variance was used for comparison among the groups. When the 1-way analysis of variance showed a significant difference $(P<.05)$, we compared the 2 groups by using the Tukey honestly significant difference test.

\section{Results}

Important preoperative and intraoperative data are shown in Table 1. There were no significant differences in the preoperative physiologic variables and the intraoperative data among the groups.

\section{Blood Biochemistry}

The preoperative biochemical parameters did not show any significant differences among the groups. Serum concentration of MDA in group $\mathrm{C}$ was significantly higher than that in the intact groups (groups A and B) at the end of ASCP $(P=.012)$. In addition, the level was significantly higher in comparison with that in groups $\mathrm{A}$ and $\mathrm{B}$, as well as that in group $\mathrm{D}$ in the rewarming phase $\left(P=.047\right.$ at $28^{\circ} \mathrm{C} ; P=$ .003 at $36^{\circ} \mathrm{C}$ ) (Figure 1). In group $\mathrm{C}$, the serum concentration of HEL became significantly higher compared with that in groups A and B, and group D at the end of ASCP, with the differences continuing to widen until the end of the experiment $(P=.005$ at the end of ASCP; $P=.0013$ at $28^{\circ} \mathrm{C} ; P=.0001$ at $36^{\circ} \mathrm{C}$ ) (Figure 2). Serum concentrations of glutamate in group $\mathrm{C}$ were significantly higher in comparison with groups $\mathrm{A}$ and $\mathrm{B}$, and group $\mathrm{D}$ during the rewarming phase $\left(P=.001\right.$ at $28^{\circ} \mathrm{C}, P=.0006$ at $\left.36^{\circ} \mathrm{C}\right)$ (Figure 3 ). In group $\mathrm{C}, \mathrm{VALD}$ was significantly greater than that in the intact groups at $28^{\circ} \mathrm{C}$ in the rewarming phase $(P=.0082)$, although the difference was not significant when compared with group D (Figure 4). However, the 2 intact groups did not differ significantly with regard to the above parameters. Likewise, values in group D were not significantly different when compared with those in the intact groups.

\section{Somatosensory Evoked Potentials}

The preoperative CCT and N4/N1 amplitude ratio did not show any difference among the groups. In group $\mathrm{C}$, the postoperative CCT was significantly prolonged compared with that in the intact groups (A and B) and group D (group A, $109 \% \pm 3.6 \%$; group B, $98.7 \% \pm 4.1 \%$; group C, $195.6 \% \pm 25.7 \%$; group $\mathrm{D}, 142.3 \% \pm 5.4 \% ; P=.0002)$ (Figure 5). Moreover, in group C, the postoperative N4/N1 amplitude ratio decreased significantly compared with that in the intact groups and group D (group A, 90.2\% $\pm 7.1 \%$; group $\mathrm{B}, 101 \% \pm 7.1 \%$; group $\mathrm{C}, 30.8 \% \pm 8.1 \%$; group $\mathrm{D}$, $60.9 \% \pm 5.3 \% ; P<.0001)$. No significant differences were noted between the 2 intact groups with regard to the postoperative CCT and N4/N1 amplitude ratio. Values in group $\mathrm{D}$ were also not significantly different compared with those in the intact groups.

\section{Discussion}

In brains with old infarction, ischemia can occur in the peri-infarct area, where the baseline blood flow is low. Such regions are known as chronic penumbra, which are nourished by collateral blood flow. ${ }^{18}$ In the present study, we maintained a cerebral perfusion flow of $10 \mathrm{~mL} / \mathrm{kg}$ per minute (perfusion pressure 40-50 $\mathrm{mm} \mathrm{Hg}$ ) during ASCP, which is consistent with our clinical practice. This perfusion flow is adequate for normal brains because they have a wider safety margin for cerebral ischemia. ${ }^{19}$ However, the 
TABLE 1. Preoperative and intraoperative data

\begin{tabular}{|c|c|c|c|c|c|}
\hline & \multicolumn{2}{|c|}{ Intact group } & \multicolumn{2}{|c|}{ Infarct group } & \multirow[b]{2}{*}{$P$ value* } \\
\hline & $\begin{array}{c}\text { Group A } \\
\text { (non-edaravone) }\end{array}$ & $\begin{array}{c}\text { Group B } \\
\text { (edaravone-treated) }\end{array}$ & $\begin{array}{c}\text { Group C } \\
\text { (non-edaravone) }\end{array}$ & $\begin{array}{c}\text { Group D } \\
\text { (edaravone-treated) }\end{array}$ & \\
\hline Age (mo) & $13.3 \pm 0.7$ & $13.7 \pm 1.0$ & $15.2 \pm 0.6$ & $15.8 \pm 0.5$ & .06 \\
\hline Body weight $(\mathrm{kg})$ & $13.8 \pm 0.7$ & $13.1 \pm 0.9$ & $13.9 \pm 0.8$ & $13.6 \pm 1.7$ & .90 \\
\hline Duration after $\mathrm{Cl}$ (mo) & - & - & $3.5 \pm 0.4$ & $4.0 \pm 0.3$ & .26 \\
\hline Preoperative Ht (\%) & $35.5 \pm 1.3$ & $33.8 \pm 1.2$ & $36.5 \pm 1.9$ & $34.3 \pm 1.3$ & .57 \\
\hline \multicolumn{6}{|l|}{$\operatorname{MAP}(\mathrm{mm} \mathrm{Hg})$} \\
\hline Preoperative & $135.3 \pm 9.3$ & $127.5 \pm 3.8$ & $134.3 \pm 3.6$ & $129.5 \pm 8.5$ & .82 \\
\hline On CPB & $76.2 \pm 4.7$ & $74.5 \pm 3.3$ & $77.2 \pm 4.2$ & $75.8 \pm 2.4$ & .97 \\
\hline Cooling $28^{\circ} \mathrm{C}$ & $66.5 \pm 3.9$ & $65.2 \pm 3.4$ & $66.8 \pm 4.3$ & $62.3 \pm 4.5$ & .86 \\
\hline Cooling $20^{\circ} \mathrm{C}$ & $59.5 \pm 2.7$ & $56.5 \pm 2.9$ & $60.2 \pm 2.8$ & $59.7 \pm 4.0$ & .84 \\
\hline ASCP $60 \mathrm{~min}$ & $43.5 \pm 2.3$ & $42.3 \pm 2.0$ & $43.0 \pm 4.6$ & $44.2 \pm 3.3$ & .98 \\
\hline End of ASCP & $49.0 \pm 5.1$ & $46.0 \pm 3.4$ & $45.8 \pm 3.8$ & $43.2 \pm 2.8$ & .77 \\
\hline Rewarming $28^{\circ} \mathrm{C}$ & $75.3 \pm 6.0$ & $74.8 \pm 5.2$ & $75.0 \pm 6.7$ & $69.5 \pm 3.2$ & .85 \\
\hline Rewarming $36^{\circ} \mathrm{C}$ & $78.2 \pm 3.8$ & $73.3 \pm 2.6$ & $74.8 \pm 5.7$ & $72.2 \pm 4.6$ & .78 \\
\hline \multicolumn{6}{|l|}{$\mathrm{PaO}_{2}(\mathrm{~mm} \mathrm{Hg})$} \\
\hline Preoperative & $106.5 \pm 7.5$ & $104.1 \pm 7.2$ & $115.8 \pm 36.2$ & $103.5 \pm 11.8$ & .84 \\
\hline On CPB & $272.3 \pm 72.4$ & $161.9 \pm 20.8$ & $214.6 \pm 50.4$ & $248.3 \pm 66.2$ & .55 \\
\hline Cooling $28^{\circ} \mathrm{C}$ & $347.4 \pm 64.1$ & $396.9 \pm 53.8$ & $469.1 \pm 61.4$ & $467.0 \pm 49.6$ & .39 \\
\hline Cooling $20^{\circ} \mathrm{C}$ & $735.2 \pm 31.2$ & $655.6 \pm 21.0$ & $562.9 \pm 77.5$ & $679.7 \pm 66.7$ & .19 \\
\hline ASCP $60 \mathrm{~min}$ & $683.1 \pm 61.9$ & $754.7 \pm 60.1$ & $792.2 \pm 32.8$ & $797.1 \pm 25.4$ & .33 \\
\hline End of ASCP & $729.7 \pm 32.7$ & $766.7 \pm 7.6$ & $688.0 \pm 28.7$ & $718.3 \pm 40.5$ & .34 \\
\hline Rewarming $28^{\circ} \mathrm{C}$ & $446.8 \pm 41.1$ & $454.8 \pm 72.1$ & $498.6 \pm 23.4$ & $466.5 \pm 21.5$ & .85 \\
\hline Rewarming $36^{\circ} \mathrm{C}$ & $200.1 \pm 53.2$ & $151.3 \pm 22.4$ & $203.8 \pm 73.9$ & $145.4 \pm 28.7$ & .75 \\
\hline \multicolumn{6}{|l|}{$\mathrm{PaCO}_{2}(\mathrm{~mm} \mathrm{Hg})$} \\
\hline Preoperative & $29.2 \pm 3.6$ & $33.1 \pm 6.5$ & $26.8 \pm 4.9$ & $36.0 \pm 8.2$ & .72 \\
\hline On CPB & $30.5 \pm 2.7$ & $32.4 \pm 1.4$ & $35.8 \pm 1.3$ & $29.4 \pm 1.4$ & .10 \\
\hline Cooling $28^{\circ} \mathrm{C}$ & $37.2 \pm 5.2$ & $41.5 \pm 1.5$ & $37.3 \pm 1.4$ & $36.4 \pm 1.7$ & .61 \\
\hline Cooling $20^{\circ} \mathrm{C}$ & $35.7 \pm 5.3$ & $41.5 \pm 2.0$ & $36.5 \pm 0.9$ & $36.7 \pm 1.6$ & .46 \\
\hline ASCP $60 \mathrm{~min}$ & $26.5 \pm 2.1$ & $30.9 \pm 2.0$ & $34.0 \pm 0.8$ & $31.3 \pm 2.9$ & .13 \\
\hline End of ASCP & $27.1 \pm 1.5$ & $31.9 \pm 0.6$ & $32.0 \pm 1.5$ & $29.1 \pm 2.5$ & .14 \\
\hline Rewarming $28^{\circ} \mathrm{C}$ & $31.2 \pm 3.9$ & $28.1 \pm 0.7$ & $28.8 \pm 2.9$ & $27.0 \pm 0.7$ & .67 \\
\hline Rewarming $36^{\circ} \mathrm{C}$ & $30.8 \pm 4.2$ & $30.5 \pm 0.4$ & $29.0 \pm 2.4$ & $27.8 \pm 2.2$ & .85 \\
\hline \multicolumn{6}{|l|}{ Arterial $\mathrm{pH}$} \\
\hline Preoperative & $7.44 \pm 0.02$ & $7.43 \pm 0.03$ & $7.46 \pm 0.02$ & $7.42 \pm 0.01$ & .48 \\
\hline On CPB & $7.38 \pm 0.03$ & $7.37 \pm 0.02$ & $7.39 \pm 0.03$ & $7.37 \pm 0.02$ & .87 \\
\hline Cooling $28^{\circ} \mathrm{C}$ & $7.35 \pm 0.03$ & $7.36 \pm 0.03$ & $7.34 \pm 0.04$ & $7.34 \pm 0.02$ & .95 \\
\hline Cooling $20^{\circ} \mathrm{C}$ & $7.37 \pm 0.03$ & $7.37 \pm 0.01$ & $7.35 \pm 0.04$ & $7.34 \pm 0.01$ & .91 \\
\hline ASCP $60 \mathrm{~min}$ & $7.37 \pm 0.02$ & $7.36 \pm 0.02$ & $7.35 \pm 0.03$ & $7.35 \pm 0.02$ & .90 \\
\hline End of ASCP & $7.36 \pm 0.02$ & $7.37 \pm 0.01$ & $7.36 \pm 0.03$ & $7.37 \pm 0.02$ & .98 \\
\hline Rewarming $28^{\circ} \mathrm{C}$ & $7.36 \pm 0.02$ & $7.34 \pm 0.01$ & $7.34 \pm 0.03$ & $7.36 \pm 0.02$ & .95 \\
\hline Rewarming $36^{\circ} \mathrm{C}$ & $7.37 \pm 0.02$ & $7.33 \pm 0.02$ & $7.35 \pm 0.02$ & $7.36 \pm 0.03$ & .88 \\
\hline \multicolumn{6}{|l|}{ Time required $(\mathrm{min})$} \\
\hline Cooling & $58.2 \pm 3.5$ & $54.8 \pm 3.8$ & $56.7 \pm 2.1$ & $58.6 \pm 4.0$ & .86 \\
\hline Rewarming & $75.6 \pm 5.3$ & $72.8 \pm 3.2$ & $72.7 \pm 3.8$ & $75.7 \pm 5.7$ & .94 \\
\hline
\end{tabular}

$\mathrm{Cl}$, Cerebral infarction; $\mathrm{Ht}$, hematocrit; $M A P$, mean artery pressure; $C P B$, cardiopulmonary bypass; $A S C P$, antegrade selective cerebral perfusion. $* 0$ ne-way analysis of variance.

flow may not be adequate for the chronic penumbra region. Ning and colleagues ${ }^{20}$ reported an accelerated free radical production in the peri-infarct region after transient focal ischemia in mice brain. Thus, it seems that brain ischemia and the consequent free radical production in the chronic penumbra region cause neurologic dysfunction after ASCP for brain with old infarction.

It is thought that hypothermic ASCP itself can also give rise to cerebral ischemia, particularly in a setting of old cerebral infarction. The cold perfusion of ASCP may cause 


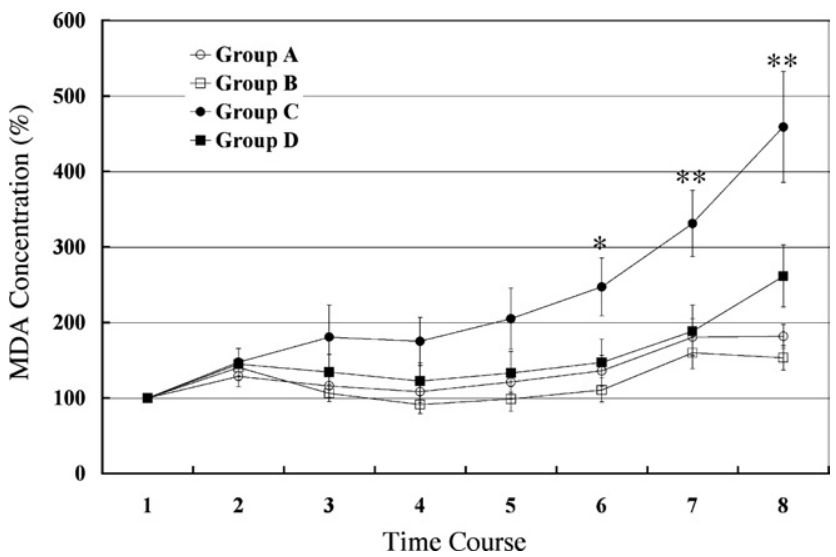

Figure 1. Changes in serum MDA concentration. Values at different time points are expressed as percentages of the preoperative value (see text). In group $C$, values significantly increased at time points 6 to 8 (*group $C$ vs groups $A$ and $B, P<.05$; ** group $C$ vs groups $A, B$, and $D, P<.05)$. MDA, Malondialdehyde.

the loss of cerebral autoregulation, ${ }^{21}$ which may result in cerebral vasoconstriction. This, in turn, may lead to a global reduction in cerebral blood flow. Moreover, phenomena such as low flow-induced astrocyte edema ${ }^{22}$ and cerebral vasospasm may also contribute to a further reduction in cerebral blood flow, particularly at the penumbral region.

Brain ischemia initiates a complex cascade of metabolic events, several of which involve the generation of nitrogen and oxygen free radicals. These free radicals and related reactive chemical species mediate much of the damage that occurs after transient brain ischemia and in the penumbral region of infarcts caused by permanent ischemia. ${ }^{23}$ Free

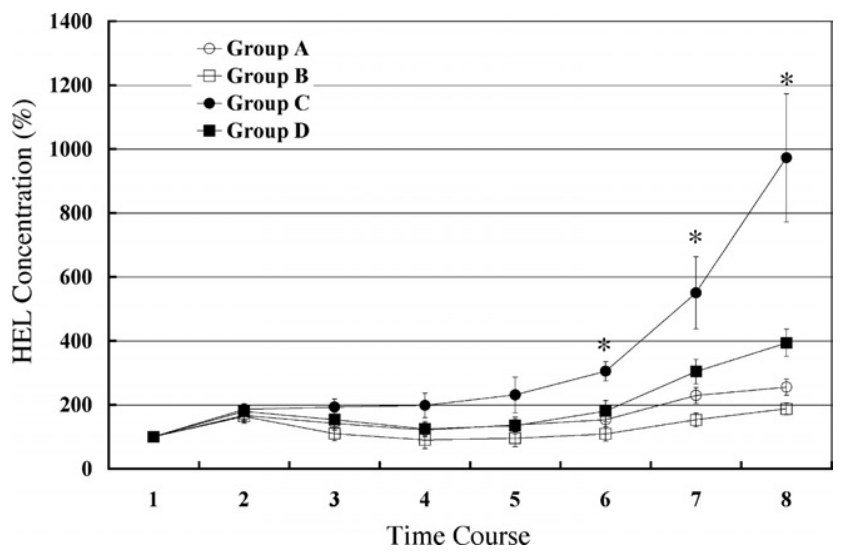

Figure 2. Changes in serum HEL concentration. Values at different time points are expressed as percentages of the preoperative value (see text). In group $C$, values significantly increased compared with that in all other groups at points 6 to 8 (*group $C$ vs groups $A, B$, and $D, P<.001)$. HEL, Hexanoyl-lysine.

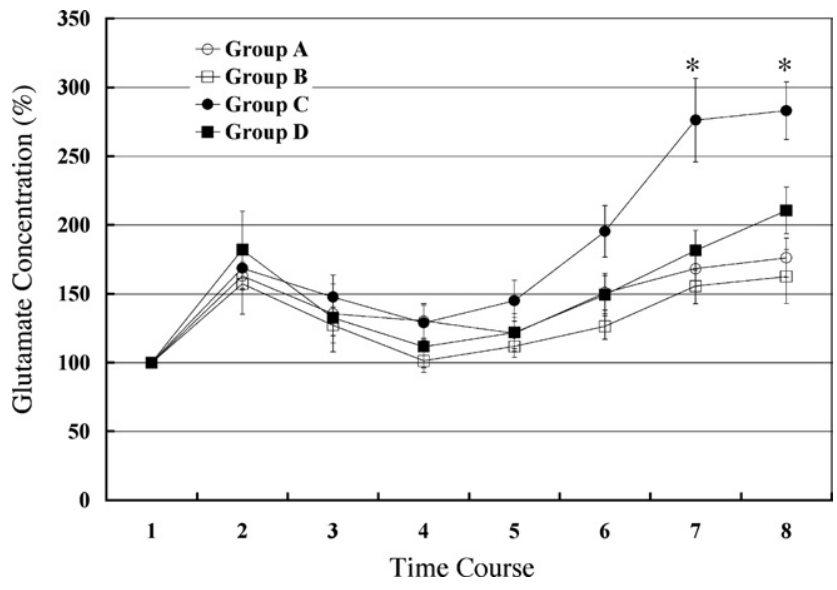

Figure 3. Changes in serum glutamate concentration. Values at different time points are expressed as percentages of the preoperative value (see text). In group C, values significantly increased compared with that in all other groups during the rewarming phase (*group C vs groups $A, B$, and $D, P<.001$ ).

radical species lead to oxidation of lipids, protein, and nucleic acid, which might alter cellular function in a critical way, resulting in neurologic injury and subsequent neurologic dysfunction. ${ }^{24,25}$ Aldehydes, such as MDA and 4-hydroxy-2-nonenal, have been well-known products of lipid peroxidation. ${ }^{14,26} \mathrm{HEL}$ is a novel lipid hydroperoxide-modified lysine residue formed in the earlier stage of lipid peroxidation by oxidative modification of linoleic acid or arachidonic acid. ${ }^{16,17}$ To our knowledge, it has not been previously used as a marker of free radical production in a cerebral infarction model. Be-

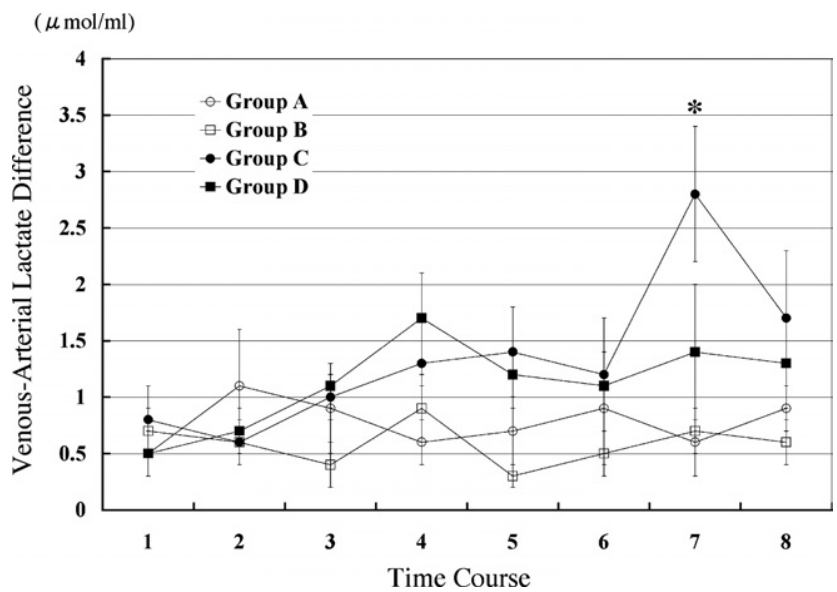

Figure 4. Level of VALD. Values are shown at different time points (see text). In group C, VALD was significantly greater than that in the intact groups at $28^{\circ} \mathrm{C}$ in the rewarming phase (*group $\mathrm{C}$ vs groups $A$ and $B, P<.001)$. VALD, Venous-arterial lactate difference. 


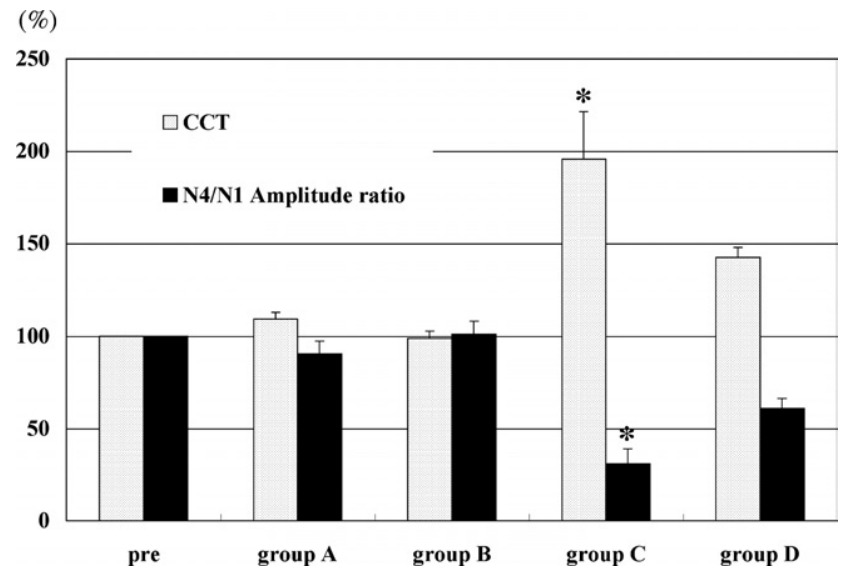

Figure 5. Changes in CCT and N4/N1 amplitude ratio. Values are expressed as percentages of the preoperative values (see text). In group C, postoperative CCT was significantly prolonged compared with that in all other groups. The postoperative N4/N1 amplitude ratio in group $C$ decreased significantly compared with that in all other groups (*group C vs groups $A, B$, and D, $P<$ .0001). CCT, Central conduction time.

cause brain is a fat-rich tissue, we believe that HEL can be a reliable biochemical marker of free radical production induced by ischemic brain injury.

In the present study, the animals in group $\mathrm{C}$ (the cerebral infarct group) showed a remarkable increase in serum HEL and MDA at the end of ASCP. These findings suggested that ASCP induced ischemia in brains with old cerebral infarction, which resulted in accelerated free radical production. Moreover, in group $\mathrm{C}$, we observed a prolongation of CCT and a decrease in the N4/N1 amplitude ratio, whereas none of these changes were seen in the intact animals. Previous studies demonstrated that impaired somatosensory evoked potential was associated with cerebral injury in permanent ischemic models. ${ }^{27,28}$ Our findings were consistent with the above findings in the sense that we also found brains with old infarction showing functional impairment with acceleration of free radical production caused by ischemia during ASCP, whereas the intact brain showed no such evidence of ischemic damage.

The animals in group D (the edaravone-treated infarct group) showed a reduction in the level of serum MDA and HEL concentrations compared with those in the non-edaravone-treated infarct animals. A decrease in postoperative CCT prolongation and the recovery of postoperative N4/N1 amplitude ratio were also noted in group D, suggesting that free radical scavenging was effective in reducing neurologic dysfunction after ASCP in brains with old infarction. Although the cerebral protective effect of edaravone in acute cerebral ischemia is well described, ${ }^{9,10,22}$ to our knowledge, this is the first report demonstrating the neuroprotective effect of edaravone during CPB for permanent cerebral infarction.

In our study, increasing free radical production was found from the point of ASCP termination until the end of the experiment in group C. Serum glutamate also showed a parallel increase during the rewarming phase in this group. In the rewarming phase, VALD also became greater in group $\mathrm{C}$, perhaps indicating an acceleration of anaerobic glycolysis in the brain tissue. ${ }^{29}$ These findings may be explained by neural cell damage being worsened by accelerated free radical production and increased cellular metabolism. It is important to note that the serum concentration of glutamate decreased in the edaravone-treated infarct group (group D) in this study. This suggests that edaravone attenuated ischemic and free radical-mediated neural cell damage and by doing so, reduced neurologic dysfunction that was documented by the results of somatosensory evoked potential examination in group D. From the above results, it is evident that in brains with old infarction, free radical scavenging provides an effective way to attenuate functional and metabolic damage to neural cells within the chronic penumbra region in the rewarming phase after ASCP.

However, the present study has a number of important limitations. Although it can be assumed from the biochemical assessment performed in the present study that brain ischemia causes free radical production in the chronic penumbra region, the actual regional cerebral blood flow during ASCP was not measured. Thus, the relationship between ischemia and free radical-mediated neurologic injury is not directly evident. Transcranial Doppler sonography or nearinfrared spectroscopy may be used to measure regional cerebral blood flow. ${ }^{30}$ Moreover, we could not perform brain histopathology to document ischemia-induced tissue damage in a graded manner.

\section{Conclusions}

We examined the influences of prophylactic administration of edaravone, a free radical scavenger, on brains with or without old cerebral infarction in dogs undergoing ASCP. Cerebral ischemia occurred during experimental ASCP resulting in postoperative neurologic dysfunction, which was attenuated by prophylactic administration of edaravone. It is therefore expected that prophylactic administration of edaravone could be useful for brain protection in ASCP for high-risk patients with a history of cerebral infarction.

\section{References}

1. Di Eusanio M, Schepens MAAM, Morshuis WJ, Di Bartolomeo R, Pierangeli A, Dossche KM. Antegrade selective cerebral perfusion during operations on the thoracic aorta: factors influencing survival and neurologic outcome in 413 patients. J Thorac Cardiovasc Surg. 2002;124:1080-6.

2. Swain JA, McDonald TJ, Griffith PK, Balaban RS, Clark RE, Ceckler T. Low-flow hypothermic CPB protects the brain. $J$ Thorac Cardiovasc Surg. 1991;102:76-84. 
3. Sukurada T, Kazui T, Tanaka H, Komatsu S. Comparative experimental study of cerebral protection during aortic arch reconstruction. Ann Thorac Surg. 1996;61:1348-54.

4. Hagl C, Ergin MA, Galla JD, Lansman SL, McCullough JN, Spielvogel D, et al. Neurologic outcome after ascending aorta-aortic arch operations: effect of brain protection technique in high risk patients. J Thorac Cardiovasc Surg. 2001;121:1107-21.

5. Kazui T, Washiyama N, Muhammad BAH, Terada H, Yamashita K, Takinami M. Improved results of atherosclerotic arch aneurysm operations with a refined technique. J Thorac Cardiovasc Surg. 2001;121: 491-9.

6. Kazui T, Washiyama N, Bashar ABH, Terada H, Yamashita K, Takimani $\mathrm{M}$, et al. Total arch replacement using aortic arch branched grafts with the aid of antegrade selective cerebral perfusion. Ann Thorac Surg. 2000;70:3-9.

7. Washiyama N, Kazui T, Takinami M, Yamashita K, Fujita S, Terada $\mathrm{H}$, et al. Experimental study on the effect of antegrade cerebral perfusion on brains with old cerebral infarction. J Thorac Cardiovasc Surg. 2001;122:734-40.

8. Ohkura K, Kazui T, Yamamoto S, Yamashita K, Tarada H, Washiyama $\mathrm{N}$, et al. Comparison of $\mathrm{pH}$ management during antegrade selective cerebral perfusion in canine models with old cerebral infarction. J Thorac Cardiovasc Surg. 2004;128:378-85.

9. Mizuno A, Umemura K, Nakashima M. Inhibitory effects of MCI-186, free radical scavenger, on cerebral ischemia following rat middle cerebral artery occlusion. Gen Pharmacol. 1998;30:575-8.

10. Edaravone Acute Infarction Study Group. Effect of a novel free radical scavenger, edaravone (MCI-186), on acute brain infarction. Randomized, placebo-controlled, double-blind study at multicenters. Cerebrovasc Dis. 2003;15:222-9.

11. Kazuchika S, Kazui T, Terada H, Umemura K, Ikeda Y, Bashar ABH, et al. Experimental study on the protective effects of edaravone against ischemic spinal cord injury. J Thorac Cardiovasc Surg. 2005;130: 1586-92.

12. Smith AL, Hoff JT, Nielsen SL, Larson CP. Barbiturate protection in acute focal cerebral ischemia. Stroke. 1974;5:1-7.

13. Takamatsu Y, Watanabe T. Studies on the concentrations of 3-methyl1-phenyl-2-pyrazolin-5-one (MCI-186) in dog plasma and cerebral spinal fluid. Jpn Pharmacol Ther. 1997;25(suppl):S1785-91.

14. Lantos J, Temes G, Roth R, Morvay G. Alterations in malondialdehyde concentration of jugular vein blood following transient brain ischemia: the effect of lactic acidosis. Acta Physiol Hung. 1994;82: 229-36.
15. Ohkawa H, Ohnishi N, Yagi K. Assay for lipid peroxides in animal tissues by thiobarbituric reaction. Anal Biochem. 1979;95:351-8.

16. Kato Y, Mori Y, Makino Y, Morimatsu Y, Hiroi S, Ishikawa T, et al Formation of $\mathrm{N} \varepsilon$-(hexanonyl)lysine in protein exposed to lipid hydroperoxide. J Biol Chem. 1999;274:20406-14.

17. Kato Y, Osawa T. Detection of lipid hydroperoxide-derived modification with polyclonal antibodies. Methods Mol Biol. 2002;186:37-44

18. Paulson OB. Regional cerebral blood flow in apoplexy due to occlusion of the middle cerebral artery. Neurology. 1970;20:63-77.

19. Tanaka H, Kazui T, Sato H, Inoue N, Yamada O, Komatsu S. Experimental study on the optimum flow rate and pressure for selective cerebral perfusion. Ann Thorac Surg. 1995;59:651-7.

20. Zhang N, Komine-Kobayashi M, Tanaka R, Liu M, Mizuno Y, Urabe $\mathrm{T}$. Edaravone reduces early accumulation of oxidative products and sequential inflammatory responses after transient focal ischemia in mice brain. Stroke. 2005;36:2220-5.

21. Watanabe T, Oshikiri N, Inui K, Kuraoka S, Minowa T, Hosaka J, et al. Optimal blood flow for cooled brain at $20^{\circ} \mathrm{C}$. Ann Thorac Surg. 1999;68:864-9.

22. Swanson RA, Ying W, Kauppinen TM. Astrocyte influence on ischemic neuronal death. Curr Mol Med. 2004;4:193-205.

23. Love S. Oxidative stress in brain ischemia. Brain Pathol. 1999;9: 119-31.

24. Lewen A, Matz P, Cfan PH. Free radical pathways in CNS injury. J Neurotrauma. 2000;17:871-90.

25. Floyd RA, Carney JM. Free radical damage to protein and DNA: mechanism involved and relevant observations on brain undergoing oxidative stress. Ann Neurol. 1992;32(suppl):S22-7.

26. Toshino H, Hattori N, Urabe T, Uchida K, Tanaka M, Mizuno Y. Postischemic accumulation of lipid peroxidation products in the rat brain: immunohistochemical detection of 4-hydroxy-2-nonenal modified proteins. Brain Res. 1997;767:81-6.

27. Meyer KL, Dempsey RJ, Roy MW, Donaldson DL. Somatosensory evoked potentials as a measure of experimental cerebral ischemia. J Neurosurg. 1985;62:269-75.

28. Skeigh JW, Havill JH, Frith R, Kersel D, Marsh N, Ulyatt D. Somatosensory evoked potentials in severe traumatic brain injury: a blinded study. J Neurosurg. 1999;91:577-80.

29. Michenfelder JD, Midle JH. The relationship among canine brain temperature, metabolism, and function during hypothermia. Anesthesiology. 1991;75:130-6

30. Yamashita K, Kazui T, Tarada H, Washiyama N, Suzuki K, Bashar AH. Cerebral oxygenation monitoring for total arch replacement using selective cerebral perfusion. Ann Thorac Surg. 2001;72:503-8. 